\title{
Lipschitz sums of convex functions
}

\author{
by \\ MARIANna CsÖRnYeI (London) and Assaf NAOR (Jerusalem)
}

\begin{abstract}
We give a geometric characterization of the convex subsets of a Banach space with the property that for any two convex continuous functions on this set, if their sum is Lipschitz, then the functions must be Lipschitz. We apply this result to the theory of $\Delta$-convex functions.
\end{abstract}

1. Introduction. This paper deals with the following problem: let $K$ be a convex subset of a Banach space $X$. Let $h_{1}, h_{2}: K \rightarrow \mathbb{R}$ be convex continuous functions such that $h_{1}+h_{2}$ is Lipschitz. Does this necessarily imply that $h_{1}$ and $h_{2}$ are themselves Lipschitz? More precisely, we are interested in the geometric properties of $K$ which imply such a statement. Under some mild assumptions, we give here a necessary and sufficient condition for $K$ to have such a property.

Throughout this paper, all Banach spaces are real. If $X$ is a Banach space, $x \in X$ and $r>0$ we use the notation $B(x, r)=\{y \in X:\|x-y\| \leq r\}$ and $S(x, r)=\{y \in X:\|x-y\|=r\}$. We also write $B_{X}=B(0,1)$ and $S_{X}=S(0,1) . X^{*}$ denotes the dual space of $X$. For any two sets $A, B$ we denote by $d(A, B)$ the distance between $A$ and $B$.

The method of proof forces us to impose some assumptions on $K$. Apart from the natural assumptions $0 \in K$ and $\overline{\operatorname{span}}(K)=X$, we essentially assume that either $K-K$ or its complement is nowhere dense. This will be formulated more precisely later, but we prefer to begin by stating the theorem in a simpler, somewhat less general form. We do not presently know if the result is true for a general convex set which is not contained in any hyperplane.

Theorem 1. Let $X$ be a Banach space and suppose that $K$ is a convex subset of $X$ for which

2000 Mathematics Subject Classification: 52A41, $26 \mathrm{~B} 25$.

Key words and phrases: convex functions, Lipschitz functions, $\Delta$-convex mappings.

Reserch of M. Csörnyei supported by the Hungarian National Foundation for Scientific Research, grant \# F029768.

Research of A. Naor partially supported by the Clore Foundation and the EU grant HPMT-CT-2000-00037. 
- $0 \in K$;

- $\overline{\operatorname{span}}(K)=X$;

and either

- $K$ is locally weakly compact

or

- K has nonempty interior.

Then the following are equivalent:

(i) If $h_{1}, h_{2}: K \rightarrow \mathbb{R}$ are continuous convex functions such that $h_{1}+h_{2}$ is Lipschitz, then both $h_{1}$ and $h_{2}$ must be Lipschitz.

(ii) There exists a constant $0<c \leq 2$ such that for every $x \in K, 0<$ $r<\operatorname{diam} K$ and $y \in S_{X}$ there are $u, v \in K \cap B(x, r)$ such that $u-v=$ cry.

(iii) There is a $c>0$ such that for any $x^{*}, y^{*} \in S_{X^{*}}$ and any $x, y \in K$ with $y^{*}(x)<y^{*}(y)$ there are $u, v \in K$ such that $y^{*}(x) \leq y^{*}(u) \leq y^{*}(v) \leq$ $y^{*}(y)$ and $\left|x^{*}(u)-x^{*}(v)\right|>c\left(y^{*}(y)-y^{*}(x)\right)$.

REMARK 2. We remark that the implication (ii) $\Rightarrow$ (i) requires only the assumptions $0 \in K$ and $\overline{\operatorname{span}}(K)=X$. Thus in our later applications, we will be able to state results for arbitrary convex subsets of Banach spaces satisfying these two assumptions.

If $K$ is bounded and has nonempty interior, then (ii) must be satisfied. Indeed, by convexity, if $B\left(x_{0}, r_{0}\right) \subset K$ for some $x_{0}$ and $r_{0}$, then $K \cap B(x, r)$ contains a segment of length $r_{0} r / \operatorname{diam} K$ in each direction. It is also easy to see that every convex set $K$ which contains an infinite open cone satisfies condition (ii).

If $\operatorname{dim} X<\infty$, then every convex set $K$ with $\overline{\operatorname{span}}(K)=X$ and $0 \in K$ has nonempty interior. It is also easy to see that if $\operatorname{dim} X<\infty$, and if a convex set $K \subset X$ has nonempty interior then conditions (i)-(iii) of Theorem 1 imply that $K$ is either bounded or contains an infinite open cone:

Corollary 3. Conditions (i)-(iii) of Theorem 1 hold for any convex set $K$ with nonempty interior, which is either bounded or contains an infinite open cone.

Suppose $\operatorname{dim} X<\infty$ and $K$ is a convex set for which $0 \in K$ and $\overline{\operatorname{span}}(K)=X$. Then (i)-(iii) of Theorem 1 are satisfied by $K$ if and only if either $K$ is bounded or $K$ contains an infinite cone $C$ for which $\overline{\operatorname{aff}}(C)=X$.

In contrast, in Remark 13 we give an example of an open unbounded convex set $K$ in an infinite-dimensional Banach space, which satisfies conditions (i)-(iii) but does not contain a half-line. We also give an example of an open convex set $K$ which contains an infinite cone $C$ such that $\overline{\operatorname{span}}(C)=X$, but for which conditions (i)-(iii) fail. 
For any convex set $K, x \in K$ and $r>0$ set

$$
K_{x, r}=K \cap B(x, r) .
$$

It is easy to see that if $K$ has nonempty interior or if $K_{x, r}$ is weakly compact for every $x \in K$ and $r>0$, then $K_{x, r}-K_{x, r}$ has nonempty interior or is weakly compact, respectively. Every weakly compact set is either nowhere dense or has nonempty interior.

In fact, our proof uses only the following fact:

(*) For every $x \in K, r>0$ and $y \notin K_{x, r}-K_{x, r}$ there exists a $z$ arbitrarily close to $y$ and there exists $z^{*} \in S_{X^{*}}$ which separates $z$ and $K_{x, r}-K_{x, r}$.

By the Hahn-Banach theorem, $(*)$ is satisfied if and only if $K_{x, r}-K_{x, r}$ is either nowhere dense or has nonempty interior. We prove, in fact, the following strengthening of Theorem 1 :

TheOREm 4. Let $X$ be a Banach space and suppose that $K$ is a convex subset of $X$ for which

- $0 \in K$;

- $\overline{\operatorname{span}}(K)=X$;

and either

- $K_{x, r}-K_{x, r}$ is nowhere dense for every $x \in K$ and $r>0$

or

- $K_{x, r}-K_{x, r}$ has nonempty interior for every $x \in K$ and $r>0$.

Then the conditions (i)-(iii) of Theorem 1 are equivalent.

A rather unexpected consequence of our result is the following fact:

COROLlary 5. Suppose that $K$ is an unbounded open set which satisfies the hypotheses of Theorem 4. If $H \subset X$ is convex and $K \subset H$, then $H$ satisfies (i).

Proof. By Remark 2, all we have to do is to check that condition (ii) for $K$ implies condition (ii) for $H$. Fix some $x \in H, r>0, y \in S_{X}$ and $z \in K$.

If $r \geq d(x, z)$ then, using (ii) for $K$, there are

$$
u, v \in B(z, r) \cap K \subset B(x, 2 r) \cap H
$$

with $u-v=$ cry. Then of course $u^{\prime}=(u+x) / 2$ and $v^{\prime}=(v+x) / 2$ are in $B(x, r) \cap H$ and $u^{\prime}-v^{\prime}=(c / 2) r y$.

On the other hand, if $r<d(x, z)$ then, using (ii) for $K$, there are

$$
u, v \in B(z, d(x, z)) \cap K \subset B(x, 2 d(x, z)) \cap H
$$

such that $u-v=c d(x, z) y$. Now, the points

$$
u^{\prime}=x+\frac{r}{2 d(x, z)}(u-x) \text { and } v^{\prime}=x+\frac{r}{2 d(x, z)}(v-x)
$$


are in $B(x, r) \cap H$ and

$$
u^{\prime}-v^{\prime}=\frac{\operatorname{crd}(x, z)}{2 d(x, z)} y=(c / 2) r y .
$$

Therefore $H$ satisfies (ii) for $c / 2$.

Before passing to the proof of Theorem 4, we discuss an application of this result to the theory of $\Delta$-convex mappings.

Let $X$ and $Y$ be Banach spaces, and $K \subset X$ a convex subset. A mapping $F: K \rightarrow Y$ is called $\Delta$-convex if there is a continuous convex function $f: K \rightarrow \mathbb{R}$ such that for every $y^{*} \in S_{Y^{*}}, y^{*} \circ F+f$ is continuous and convex. Such an $f$ is called a control function for $F$.

Such functions are known to have many interesting properties. For instance, it is known that the $\Delta$-convex mappings from $\mathbb{R}^{n}$ to $\mathbb{R}$ form an algebra, and that the composition of two $\Delta$-convex mappings is itself $\Delta$ convex. $\Delta$-convex mappings have applications in optimization theory, approximation theory, and the theory of Gateaux differentiability of convex functions. For more information we refer to the papers [VZ], [DVZ] and the references therein. The geometric importance of $\Delta$-convex mappings may be appreciated from a result of $\mathrm{M}$. Cepedello Boiso [C1, C2] (see also the book $[\mathrm{BL}]$ ), which states that a Banach space $X$ is superreflexive if and only if every real-valued Lipschitz function on $X$ can be uniformly approximated by $\Delta$-convex functions.

The properties of a control function $f$ for a $\Delta$-convex function $F$ impose restrictions on the behavior of $F$. For instance, it is proved in [VZ] that $F$ is differentiable (Fréchet or Gateaux) at some point whenever $f$ is. One can ask: is $F$ Lipschitz whenever $f$ is? Theorem 1 and a simple Banach-Steinhaus argument give the following improvement of Theorem 18 in [DVZ]:

Theorem 6. Let $X$ and $Y$ be Banach spaces and $K \subset X$ a convex set satisfying (ii). If $F: K \rightarrow Y$ is $\Delta$-convex with Lipschitz control function $f$, then $F$ is Lipschitz.

Proof. For each $y^{*} \in S_{Y^{*}}$, the functions $g_{1}=y^{*} \circ F+f$ and $g_{2}=$ $-y^{*} \circ F+f$ are continuous and convex on $K$, and $g_{1}+g_{2}$ is Lipschitz. By Remark 2, $K$ also satisfies (i), so that $g_{1}$ and $g_{2}$ are Lipschitz. Consequently, $y^{*} \circ F$ is Lipschitz for each $y^{*} \in Y^{*}$. The Banach-Steinhaus theorem now implies that $F$ is Lipschitz.

The example in Remark 13 shows that this is a strict improvement of the result of [DVZ].

Acknowledgment. We would like to express our sincere gratitude to Libor Veselý for suggesting the problem to us and pointing out that our results imply Theorem 6 . 
2. Proof of Theorem 4. Let $K \subset X$ be a convex set which satisfies the assumptions of Theorem 4 . We break the proof of Theorem 4 down into several lemmas. We prove the theorem by showing that (ii) $\Rightarrow($ i), (iii) $\Rightarrow$ (ii) and $\neg($ iii $) \Rightarrow \neg$ (i).

For every pair of vectors $x, y \in X \backslash\{0\}$ define

$$
\alpha(0, x, y)=\min (d(x /\|x\|, \mathbb{R} y), d(y /\|y\|, \mathbb{R} x)) .
$$

For any $p \in X \backslash\{x, y\}$ put $\alpha(p, x, y)=\alpha(0, x-p, y-p)$. Similarly, if $x, y \in X$ and $p \in X \backslash\{x, y\}$ then we define

$$
\beta(p, x, y)=\frac{\|x-y\|}{\max (\|p-x\|,\|p-y\|)} .
$$

Obviously $\beta(p, x, y) \geq \alpha(p, x, y)$.

We begin by showing that condition (ii) of Theorem 4 implies an apparently stronger geometric condition.

Lemma 7. Assume that condition (ii) of Theorem 4 holds for some constant $0<c \leq 2$. Then for every $x \in K, r<\operatorname{diam} K$ and $y \in S_{X}$ there are $u, v \in K \cap B(x, r)$ such that:

(1) $u-v=c^{2} r y /(8+c)$;

(2) $d(x+\mathbb{R} y, u+\mathbb{R} y) \geq c r / 10$;

(3) $\beta(x, u, v) \geq \alpha(x, u, v) \geq c^{3} / 120$.

Proof. Define $r^{\prime}=r /(1+c / 8)$. Note that there is some $z \in K \cap B\left(x, r^{\prime}\right)$ such that $d(z, x+\mathbb{R} y) \geq c r^{\prime} / 4$. Indeed, if $y^{*}$ is a norm-one functional with $y^{*}(y)=0$ then for every $w \in S_{X}$ with $y^{*}(w)>1 / 2$, by our assumption, there are $a, b \in K \cap B\left(x, r^{\prime}\right)$ for which $a-b=c r^{\prime} w$. Then

$$
d(a, x+\mathbb{R} y)+d(b, x+\mathbb{R} y) \geq\left|y^{*}(a-x)\right|+\left|y^{*}(b-x)\right| \geq y^{*}\left(c r^{\prime} w\right)>c r^{\prime} / 2,
$$

thus $\max \{d(a, x+\mathbb{R} y), d(b, x+\mathbb{R} y)\} \geq c r^{\prime} / 4$.

Fix any such $z$. Using the assumption again, there are

$$
u, v \in K \cap B\left(z, \frac{c r^{\prime}}{8}\right) \subset K \cap B(x, r)
$$

for which

$$
u-v=c \frac{c r^{\prime}}{8} y=\frac{c^{2} r}{8+c} y
$$

Now

$$
d(x+\mathbb{R} y, u+\mathbb{R} y) \geq \frac{c r^{\prime}}{4}-\frac{c r^{\prime}}{8}=\frac{c r}{8+c} \geq \frac{c r}{10} .
$$

For any $q \in x+\mathbb{R}(u-x)$ write $q=\lambda u+(1-\lambda) x$ and define

$$
q^{\prime}=x+\frac{v-q}{1-\lambda}=u+\frac{v-u}{1-\lambda} .
$$


Clearly $q^{\prime}$ is on the line $u+\mathbb{R} y$, thus $\left\|q^{\prime}-x\right\| \geq c r / 10$. Therefore

$$
\|v-q\|=\left\|q^{\prime}-x\right\||1-\lambda|=\left\|q^{\prime}-x\right\| \cdot \frac{\|u-q\|}{\|u-x\|} \geq \frac{c r}{10} \cdot \frac{\|u-v\|-\|v-q\|}{\|u-x\|} .
$$

Hence

$$
\|v-q\| \geq \frac{(c r / 10) \cdot\|u-v\|}{\|u-x\|+c r / 10} \geq \frac{(c r / 10) \cdot\left(c^{2} r / 10\right)}{r+c r / 10} \geq \frac{c^{3} r}{120} .
$$

Finally,

$$
\frac{\|v-q\|}{\|v-x\|} \geq \frac{c^{3}}{120}
$$

which easily implies that

$$
d\left(\frac{v-x}{\|v-x\|}, \mathbb{R}(u-x)\right) \geq \frac{c^{3}}{120} .
$$

Then (3) follows by symmetry.

Lemma 8. Assume that $f, g: K \rightarrow \mathbb{R}$ are such that $h_{1}=g+f$ and $h_{2}=$ $g-f$ are continuous convex functions, and $g$ is Lipschitz with constant $L$. Then for every line $\ell$ which intersects $K$ in at least two points and for every $x, y, z, w \in \ell \cap K$ with $x \neq y, z \neq w$,

$$
\frac{|f(x)-f(y)|}{\|x-y\|}-\frac{|f(z)-f(w)|}{\|z-w\|} \leq 4 L .
$$

Proof. Without loss of generality we may assume that $\ell=\mathbb{R}$. Put $u=$ $\min (x, y, z, w)$ and $v=\max (x, y, z, w)$. Then for any $a, b \in[u, v]$ with $a \neq b$ and $i=1,2$,

$$
D^{+} h_{i}(u) \leq \frac{h_{i}(a)-h_{i}(b)}{a-b} \leq D^{-} h_{i}(v),
$$

where $D^{+}$and $D^{-}$denote the right and left derivatives, respectively. In particular, $0 \leq D^{-} h_{i}(v)-D^{+} h_{i}(u)$, and so

$$
\begin{aligned}
0 & \leq\left(D^{-} h_{2}(v)-D^{+} h_{2}(u)\right)+\left(D^{-} h_{1}(v)-D^{+} h_{1}(u)\right) \\
& =2 D^{-} g(v)-2 D^{+} g(u) \leq 4 L .
\end{aligned}
$$

It follows that either $0 \leq D^{-} h_{1}(v)-D^{+} h_{1}(u) \leq 2 L$, or $0 \leq D^{-} h_{2}(v)-$ $D^{+} h_{2}(u) \leq 2 L$. If $0 \leq D^{-} h_{1}(v)-D^{+} h_{1}(u) \leq 2 L$, then

$$
\begin{aligned}
4 L & \geq\left(D^{-} h_{1}(v)+L\right)-\left(D^{+} h_{1}(u)-L\right) \\
& \geq \frac{\left(h_{1}(x)-h_{1}(y)\right)-(g(x)-g(y))}{x-y}-\frac{\left(h_{1}(z)-h_{1}(w)\right)-(g(z)-g(w))}{z-w} \\
& =\frac{f(x)-f(y)}{x-y}-\frac{f(z)-f(w)}{z-w} .
\end{aligned}
$$

If $0 \leq D^{-} h_{2}(v)-D^{+} h_{2}(u) \leq 2 L$, then a similar calculation gives the same bound. 
Proof that (ii) $\Rightarrow$ (i). Suppose that $h_{1}, h_{2}$ are continuous convex functions on $K$ for which $h_{1}+h_{2}$ is Lipschitz. We write $h_{1}=g+f$ and $h_{2}=g-f$. Then $g$ is Lipschitz with constant $L$, say. Our goal is to prove that $f$ is also Lipschitz.

Fix two points $p_{1}, p_{2} \in K$ and some $\varepsilon>0$. Since $f$ is continuous at $p_{1}$ and $p_{2}$, there is a $0<\delta \leq\left\|p_{1}-p_{2}\right\| / 4$ such that $\left|f\left(p_{1}\right)-f(q)\right|<\varepsilon$ for every $q \in B\left(p_{1}, \delta\right)$ and $\left|f\left(p_{2}\right)-f(q)\right|<\varepsilon$ for every $q \in B\left(p_{2}, \delta\right)$. Let $\ell$ be a line which intersects $K$ in at least two points. Take any $p$ which is in the relative interior of the interval $K \cap \ell$. Clearly $\max \left\{\left\|p-p_{1}\right\|,\left\|p-p_{2}\right\|\right\} \geq 2 \delta$, so that we may assume that $\left\|p-p_{1}\right\|=d \geq 2 \delta$.

Applying Lemma 7 for $x=p, r=d-\delta$ and $y$ a unit vector in the direction of $\ell$, we find $u, v \in K \cap B(p, d-\delta)$ which satisfy conclusions (1)-(3) of Lemma 7. For every $a \in B(p, d-\delta) \cap K$ we have $\left\|p_{1}-a\right\| \geq \delta$. By convexity, this implies that $a^{\prime}=p_{1}+\delta\left(a-p_{1}\right) /\left\|a-p_{1}\right\|$ is in $K$. Lemma 8 applied to the line connecting $a$ and $p_{1}$ shows that for any two points $b, c$ on this line,

$$
\frac{|f(b)-f(c)|}{\|b-c\|} \leq \frac{\left|f\left(a^{\prime}\right)-f\left(p_{1}\right)\right|}{\left\|a^{\prime}-p_{1}\right\|}+4 L \leq \frac{\varepsilon}{\delta}+4 L .
$$

By Lemma 7 the distances between $p$ and $u$ and between $p$ and $v$ are at least $c(d-\delta) / 10 \geq c d / 20$. On the other hand, the distance between $p_{1}$ and any of the points $p, u, v$ is at most $2 d$. Hence

$$
\begin{aligned}
|f(u)-f(p)| & \leq\left|f(u)-f\left(p_{1}\right)\right|+\left|f(p)-f\left(p_{1}\right)\right| \leq\left(\frac{\varepsilon}{\delta}+4 L\right) \cdot 4 d \\
& \leq\left(\frac{\varepsilon}{\delta}+4 L\right) \cdot \frac{80\|u-p\|}{c}
\end{aligned}
$$

That is,

$$
\frac{|f(u)-f(p)|}{\|u-p\|} \leq \frac{80}{c}\left(\frac{\varepsilon}{\delta}+4 L\right),
$$

and similarly,

$$
\frac{|f(v)-f(p)|}{\|v-p\|} \leq \frac{80}{c}\left(\frac{\varepsilon}{\delta}+4 L\right)
$$

In addition, we know that $\alpha(p, u, v) \geq c^{3} / 120$.

Now we use the continuity of $f$ to find some $p^{\prime}=p+\lambda y \in K \cap \ell$ where $\lambda>0$ is small enough to ensure that

$$
\max \left(\frac{\left|f(u)-f\left(p^{\prime}\right)\right|}{\left\|u-p^{\prime}\right\|}, \frac{\left|f(v)-f\left(p^{\prime}\right)\right|}{\left\|v-p^{\prime}\right\|}\right) \leq \frac{100}{c}\left(\frac{\varepsilon}{\delta}+4 L\right) .
$$

Let $s$ be the intersection point of the line segments between $p$ and $u$, and $p^{\prime}$ and $v$ (note that $p^{\prime}-p$ and $u-v$ have the same direction, therefore this 
intersection point exists). By Lemma 8,

$$
\frac{|f(p)-f(s)|}{\|p-s\|} \leq \frac{|f(p)-f(u)|}{\|p-u\|}+4 L \leq \frac{100}{c}\left(\frac{\varepsilon}{\delta}+5 L\right),
$$

and similarly for $p^{\prime}$ replacing $p$ and $v$ replacing $u$. Hence if $\lambda$ is small enough then

$$
\frac{\left|f\left(p^{\prime}\right)-f(s)\right|}{\left\|p^{\prime}-s\right\|} \leq \frac{100}{c}\left(\frac{\varepsilon}{\delta}+6 L\right) .
$$

By choosing $\lambda$ to be small enough, we can also ensure that $\alpha(s, u, v)=$ $\alpha\left(s, p, p^{\prime}\right) \geq c^{3} / 200$. Hence

$$
\begin{aligned}
\left|f(p)-f\left(p^{\prime}\right)\right| & \leq|f(p)-f(s)|+\left|f\left(p^{\prime}\right)-f(s)\right| \\
& \leq 2 \max \left(\|p-s\|,\left\|p^{\prime}-s\right\|\right) \cdot \frac{100}{c} \cdot\left(\frac{\varepsilon}{\delta}+6 L\right) \\
& =\frac{\left\|p-p^{\prime}\right\|}{\beta\left(s, p, p^{\prime}\right)} \cdot \frac{200}{c} \cdot\left(\frac{\varepsilon}{\delta}+6 L\right) \leq \frac{\left\|p-p^{\prime}\right\|}{\alpha\left(s, p, p^{\prime}\right)} \cdot \frac{200}{c} \cdot\left(\frac{\varepsilon}{\delta}+6 L\right) \\
& \leq \frac{40000\left\|p-p^{\prime}\right\|}{c^{4}}\left(\frac{\varepsilon}{\delta}+6 L\right) .
\end{aligned}
$$

This proves that $f$ is Lipschitz on each line $\ell$ with Lipschitz constant

$$
\frac{40000}{c^{4}}\left(\frac{\varepsilon}{\delta}+6 L\right)
$$

Proof that (iii) $\Rightarrow$ (ii). It is convenient to distinguish three cases:

CASE 1: $K$ is bounded.

CAse 2: $K$ is unbounded, and there is an $x^{*} \in S_{X^{*}}$ for which

$$
\sup _{x \in K}\left|x^{*}(x)\right|<\infty .
$$

Case 3: For every $x^{*} \in S_{X^{*}}$ we have

$$
\sup _{x \in K}\left|x^{*}(x)\right|=\infty .
$$

Proof for Case 1. In this case condition (ii) of Theorem 4 is equivalent to the fact that for some $\varepsilon>0, K$ contains a line segment of length $\varepsilon$ in each direction. Indeed, (ii) clearly implies the existence of such line segments. On the other hand, if there are segments of length $\varepsilon$ in each direction, then by convexity for every $x \in K$ and $r<\operatorname{diam} K$ the ball $B(x, r)$ contains a segment of length $\varepsilon r / \operatorname{diam} K$ in every direction.

Fix any $y^{*} \in S_{X^{*}}$ and $x, y \in K$ such that $y^{*}(x)<y^{*}(y)$. Assume that (ii) does not hold. Then there are unit vectors $\left\{y_{n}\right\}_{n=1}^{\infty} \subset S_{X}$ and a sequence $\left\{\varepsilon_{n}\right\}_{n=1}^{\infty}$ of positive numbers tending to zero such that $\varepsilon_{n} y_{n} \notin K-K$. Our assumption $(*)$ on $K$ ensures that there is a $z_{n}$ of norm arbitrarily close to $\varepsilon_{n}$ which can be separated from $K-K$. Therefore there is an $x_{n}^{*} \in S_{X^{*}}$ 
such that $x_{n}^{*}(u)-x_{n}^{*}(v) \leq 3 \varepsilon_{n}$ for all $u, v \in K$. But condition (iii) implies the existence of $u, v \in K$ for which

$$
c\left(y^{*}(y)-y^{*}(x)\right)<x_{n}^{*}(u)-x_{n}^{*}(v) \leq 3 \varepsilon_{n},
$$

which is a contradiction when $n$ is large enough.

Proof for Case 2. In this case it is easy to see that (iii) cannot hold. Indeed, since $K$ is unbounded, we can find $y^{*} \in S_{X^{*}}$ for which $\sup _{x \in K}\left|y^{*}(x)\right|$ $=\infty$. Then for every $c>0$ there are $x, y \in K$ such that

$$
c\left(y^{*}(y)-y^{*}(x)\right)>2 \sup _{x \in K}\left|x^{*}(x)\right| \geq \sup _{u, v \in K}\left|x^{*}(u)-x^{*}(v)\right| .
$$

Proof for Case 3. Assume first that (ii) fails for some constant $0<c<$ $1 / 3$. In other words, there are $0<c<1 / 3, x \in K, y \in S_{X}$ and $r>0$ for which

$$
c r y \notin K_{x, r}-K_{x, r} .
$$

Without loss of generality, we may assume that $x=0$. Hence, for every $\varepsilon>0$ we can find $z^{*} \in S_{X^{*}}$ such that $z^{*}(u)-z^{*}(v) \leq(c+\varepsilon) r$ for all $u, v \in K \cap B(0, r)$, that is, by choosing $\varepsilon<1 / 3-c$ and by replacing $c+\varepsilon$ by $c$ we find $z^{*} \in S_{X^{*}}$ and $c \in(0,1 / 3)$ such that $z^{*}(u)-z^{*}(v) \leq c r$ for all $u, v \in K \cap B(0, r)$. In particular, for every $u \in K \cap B(0, r)$ we have $\left|z^{*}(u)\right| \leq c r$. Define

$$
\begin{aligned}
& A^{+}=\left\{x \in X: z^{*}(x)>c r \text { and } z^{*}(x)>c\|x\|\right\}, \\
& A^{-}=\left\{x \in X: z^{*}(x)<-c r \text { and } z^{*}(x)<-c\|x\|\right\} .
\end{aligned}
$$

We claim that both $A^{+}$and $A^{-}$are disjoint from $K$. Indeed, if $z^{*}(x)>c r$ and $z^{*}(x)>c\|x\|$ for some $x \in K$, then there is a $\lambda>1$ such that $z^{*}(x)>\lambda c r$ and $z^{*}(x)>\lambda c\|x\|$. Then $\lambda c r x /\left|z^{*}(x)\right| \in K \cap B(0, r)$, so $\left|z^{*}\left(\lambda c r x /\left|z^{*}(x)\right|\right)\right| \leq$ $c r$, which is a contradiction. Similarly for $A^{-}$.

By the Hahn-Banach theorem, since $A^{+}, A^{-}$are convex with nonempty interior, we can find $x^{*}, v^{*} \in S_{X^{*}}$ and $\alpha \geq 0 \geq \beta$ for which

$$
\inf _{u \in A^{+}} x^{*}(u) \geq \alpha \geq \sup _{u \in K} x^{*}(u), \quad \inf _{u \in K} v^{*}(u) \geq \beta \geq \sup _{u \in A^{-}} v^{*}(u) .
$$

Since $x^{*}, v^{*}$ are unbounded on $K$ by our assumption, we have $x^{*} \neq v^{*}$. Let $\delta>0$, and take $x, z \in B(0,1+\delta)$ for which $x^{*}(x)=z^{*}(z)=1$. Note that $z^{*}(u) \leq c\|u\|$ for any $u \in \operatorname{ker}\left(x^{*}\right)$, since otherwise for $\lambda$ large enough and $u^{\prime}=\lambda u \in \operatorname{ker}\left(x^{*}\right)$ we would have $u^{\prime} \in \operatorname{int}\left(A^{+}\right)$, a contradiction. Since $z=x^{*}(z) x+\left(z-x^{*}(z) x\right)$ and $z-x^{*}(z) x \in \operatorname{ker}\left(x^{*}\right)$, for $\delta$ sufficiently small we have

$$
\begin{aligned}
1 & =z^{*}(z)=x^{*}(z) z^{*}(x)+z^{*}\left(z-x^{*}(z) x\right) \\
& \leq x^{*}(z) z^{*}(x)+c\left\|z-x^{*}(z) x\right\| \leq x^{*}(z) z^{*}(x)+3 c .
\end{aligned}
$$


It is also easy to see that $\lambda z \in A^{+}$if $\lambda$ is large enough and $\delta$ is small enough, therefore $x^{*}(z) \geq 0$. Then, since $c<1 / 3$ and $1 \leq x^{*}(z) z^{*}(x)+3 c$, it is immediate to see that $x^{*}(z)>0$ and $z^{*}(x)>0$. Therefore

$$
1 \leq x^{*}(z) z^{*}(x)+3 c \leq(1+\delta) z^{*}(x)+3 c,
$$

that is, $z^{*}(x) \geq(1-3 c) /(1+\delta)>1-4 c$ if $\delta$ is small enough. Hence, for any $u \in S_{X}$,

$$
\begin{aligned}
c(2+\delta) & \geq c\left\|u-x^{*}(u) x\right\| \geq\left|z^{*}\left(u-x^{*}(u) x\right)\right|=\left|z^{*}(u)-x^{*}(u) z^{*}(x)\right| \\
& =\left|z^{*}(u)-x^{*}(u)+x^{*}(u)\left(1-z^{*}(x)\right)\right| \geq\left|z^{*}(u)-x^{*}(u)\right|-4 c .
\end{aligned}
$$

Thus $\left\|z^{*}-x^{*}\right\| \leq c(6+\delta)$ for every $\delta$ small enough, therefore $\left\|z^{*}-x^{*}\right\| \leq 6 c$. Similarly, we find that $\left\|z^{*}-v^{*}\right\| \leq 6 c$.

Let

$$
c_{0}=\left\|x^{*}-v^{*}\right\| \leq 12 c,
$$

take an arbitrary point $x_{1} \in K$, and define

$$
y^{*}=\frac{x^{*}-v^{*}}{c_{0}} \in S_{X^{*}} .
$$

By assumption, no functional is bounded on $K$, and yet

$$
y^{*}(x) \leq \frac{\alpha-\beta}{c_{0}} \quad \forall x \in K
$$

Therefore for every $t<y^{*}\left(x_{1}\right)$ we can find an $x_{2} \in K$ for which $y^{*}\left(x_{2}\right)=t$. Fix $x_{2}$ with

$$
y^{*}\left(x_{2}\right)=2 y^{*}\left(x_{1}\right)-\frac{\alpha-\beta}{c_{0}} .
$$

Since $v^{*}(x) \geq \beta$, we have $x^{*}(x) \geq \beta+x^{*}\left(x_{2}\right)-v^{*}\left(x_{2}\right)$ for any $x \in K$ for which $y^{*}\left(x_{2}\right) \leq y^{*}(x)$. Thus, for any $y_{1}, y_{2} \in K$ for which $y^{*}\left(x_{2}\right) \leq y^{*}\left(y_{i}\right) \leq y^{*}\left(x_{1}\right)$, we see that $\alpha \geq x^{*}\left(y_{i}\right) \geq \beta+x^{*}\left(x_{2}\right)-v^{*}\left(x_{2}\right)$. That is,

$$
\begin{aligned}
\left|x^{*}\left(y_{1}\right)-x^{*}\left(y_{2}\right)\right| & \leq \alpha-\beta-x^{*}\left(x_{2}\right)+v^{*}\left(x_{2}\right)=\alpha-\beta-y^{*}\left(x_{2}\right) c_{0} \\
& =\left(2 y^{*}\left(x_{1}\right)-y^{*}\left(x_{2}\right)\right) c_{0}-y^{*}\left(x_{2}\right) c_{0} \\
& =2 c_{0}\left(y^{*}\left(x_{1}\right)-y^{*}\left(x_{2}\right)\right) \\
& \leq 24 c\left(y^{*}\left(x_{1}\right)-y^{*}\left(x_{2}\right)\right) .
\end{aligned}
$$

Summarizing, we have proved that if condition (iii) is satisfied with a constant $c$, then condition (ii) must be satisfied with constant $c / 24$, provided that $c / 24<1 / 3$. But if (iii) is satisfied for some $c$ then it is also satisfied for every $c^{\prime}<c$, which completes our proof.

This finishes the proof of the implication (iii) $\Rightarrow$ (ii).

For later purposes, we record here that from the proof of Case 2 it follows that if $K$ is unbounded and (iii) fails for some constant $c$, then there are 
$x^{*}, y^{*} \in S_{X^{*}}$ and $x, y \in K$ such that (iii) fails for $c, x, y, x^{*}, y^{*}$ and $y^{*}$ is not bounded on $K$. By symmetry, we can assume that $\sup _{x \in K} y^{*}(x)=\infty$.

It remains to prove that if (iii) fails then there are continuous functions $f, g: K \rightarrow \mathbb{R}$ such that $f$ is not Lipschitz, $g$ is Lipschitz and $h_{1}=g+f$, $h_{2}=g-f$ are convex.

We begin by defining some auxiliary functions:

Lemma 9. Fix $0<\varepsilon<1 / 2, \beta>0$ and $L>1$. Let $\alpha=\varepsilon^{2} \beta$ and $\gamma=e^{L} \beta$. Then there are continuous functions $f, g: \mathbb{R}^{2} \rightarrow \mathbb{R}$ for which

(A) $g+f, g-f$ are both convex on $([-\alpha, \alpha] \times \mathbb{R}) \cup(\mathbb{R} \times(\mathbb{R} \backslash[0,2 \gamma]))$;

(B) $\left|f_{y}\right|<\varepsilon$ and $\left|g_{x}\right|,\left|g_{y}\right|<40 \varepsilon$ on $([-\alpha, \alpha] \times \mathbb{R}) \cup(\mathbb{R} \times(\mathbb{R} \backslash[0,2 \gamma]))$;

(C) $|f(x, y)| \leq \alpha L$ on $[-\alpha, \alpha] \times \mathbb{R}$ and $f(x, y)=0$ on $\mathbb{R} \times(\mathbb{R} \backslash[\beta, 2 \gamma-\beta])$;

(D) $f_{x}(x, \gamma)=L$ and $\left|f_{x}(x, y)\right| \leq L$ for every $x, y \in \mathbb{R}$.

(Here $f_{x}, f_{y}, g_{x}, g_{y}$ denote the partial derivatives of $f$ and $g$.)

Proof. Denote the sets $\{y \leq \beta\},\{\beta \leq y \leq \gamma\},\{\gamma \leq y \leq 2 \gamma-\beta\}$ and $\{2 \gamma-\beta \leq y\}$ by $S_{1}, S_{2}, S_{3}$ and $S_{4}$, respectively. Let

$$
f(x, y)= \begin{cases}0, & y \in S_{1}, \\ x \log (y / \beta), & y \in S_{2},\end{cases}
$$

and extend $f$ to $\mathbb{R} \times S_{3}$ and $\mathbb{R} \times S_{4}$ by $f(x, y)=f(x, 2 \gamma-y)$. Clearly (C) and (D) hold. Let $g=p+q+r$, where

$$
\begin{aligned}
& p(x, y)= \begin{cases}0, & y+|x| \leq \beta, \\
\varepsilon(y+|x|-\beta)^{2} / \alpha, & y \leq \beta \leq y+|x|, \\
\varepsilon x^{2} / \alpha, & y \in S_{2},\end{cases} \\
& q(x, y)= \begin{cases}0, & y \in S_{1}, \\
-\alpha \log (y / \beta) / \varepsilon, & y \in S_{2},\end{cases} \\
& r(x, y)= \begin{cases}0, & y \in S_{1}, \\
12 \varepsilon(y-\beta), & y \in S_{2}, \\
12 \varepsilon(2 y-\beta-\gamma), & y \in S_{3}, \\
12 \varepsilon(3 y-3 \gamma), & y \in S_{4},\end{cases}
\end{aligned}
$$

and extend $p, q$ to the whole plane by $p(x, y)=p(x, 2 \gamma-y), q(x, y)=$ $q(x, 2 \gamma-y)$. These are well defined continuous functions.

It is easy to check that the restriction of $g$ to $\mathbb{R} \times S_{1}$ is convex, and thus, as the restriction of $f$ to $\mathbb{R} \times S_{1}$ is 0 , it follows that $g+f$ and $g-f$ are convex on $\mathbb{R} \times S_{1}$. It is also straightforward to verify that the restrictions of $g+f$ and $g-f$ to $\left\{-\alpha \leq x \leq \alpha, y \in S_{2}\right\}$ are convex: Indeed, on this set $g+f$ and $g-f$ may be written as the sum of linear functions together with the functions $h^{+}$and $h^{-}$, respectively, where $h^{ \pm}(x, y)= \pm x \log y+\varepsilon x^{2} / \alpha-(\alpha \log y) / \varepsilon$. On calculating the second partial derivatives of $h^{ \pm}$we find $h_{x x}^{ \pm}=2 \varepsilon / \alpha>0$ 
and

$$
h_{x x}^{ \pm} h_{y y}^{ \pm}-\left(h_{x y}^{ \pm}\right)^{2}=\frac{2 \varepsilon(\alpha / \varepsilon \mp x) / \alpha-1}{y^{2}}=\frac{2 \mp 2 \varepsilon x / \alpha-1}{y^{2}} \geq \frac{1-2 \varepsilon}{y^{2}}>0,
$$

that is, the functions $h^{ \pm}$are convex on $\mathbb{R} \times S_{2}$. This also shows that $g+f$ and $g-f$ are convex on the sets $\mathbb{R} \times S_{4}$ and $\left\{-\alpha \leq x \leq \alpha, y \in S_{3}\right\}$. Therefore, in order to show that $g \pm f$ are convex on $([-\alpha, \alpha] \times \mathbb{R}) \cup(\mathbb{R} \times(\mathbb{R} \backslash[0,2 \gamma]))$, it only remains to check their convexity around the points of the segments $\{y=\beta\},\{y=\gamma\}$ and $\{y=2 \gamma-\beta\}$.

At the points $(x, y) \in([-\alpha, \alpha] \times \mathbb{R}) \cup(\mathbb{R} \times(\mathbb{R} \backslash[0,2 \gamma]))$ we have

$$
\begin{gathered}
f_{x}(x, y)=\left\{\begin{array}{ll}
0, & y \in S_{1}, \\
\log (y / \beta), & y \in S_{2},
\end{array} \quad\left|f_{y}(x, y)\right| \leq \frac{\alpha}{\beta}<\varepsilon, \quad\left|p_{x}\right|,\left|p_{y}\right| \leq 2 \varepsilon,\right. \\
q_{x}=r_{x}=0, \quad\left|q_{y}\right| \leq \frac{\alpha}{\varepsilon \beta}=\varepsilon, \quad r_{y}= \begin{cases}0, & y \in S_{1}, \\
12 \varepsilon, & y \in S_{2}, \\
24 \varepsilon, & y \in S_{3}, \\
36 \varepsilon, & y \in S_{4} .\end{cases}
\end{gathered}
$$

Since $\left|f_{y}\right|,\left|p_{y}\right|,\left|q_{y}\right|$ are at most $2 \varepsilon$ each, and $\left.r_{y}\right|_{S_{i+1}}-\left.r_{y}\right|_{S_{i}}=12 \varepsilon$, it follows that

$$
\left.\left(g_{y} \pm f_{y}\right)\right|_{S_{i+1}}-\left.\left(g_{y} \pm f_{y}\right)\right|_{S_{i}} \geq 0 .
$$

From this it easily follows that $g \pm f$ are also convex around the points of the required segments. This also shows that (B) is satisfied.

Proof that (i) $\Rightarrow($ iii). Suppose that (iii) does not hold. For every $n$ fix $c_{n}>0, x_{n}, y_{n} \in K$ and $x_{n}^{*}, y_{n}^{*} \in S_{X^{*}}$ such that $c_{n} \rightarrow 0, y_{n}^{*}\left(x_{n}\right)<y_{n}^{*}\left(y_{n}\right)$ and for every $n$ and $u, v \in K$ with $y_{n}^{*}\left(x_{n}\right) \leq y_{n}^{*}(u) \leq y_{n}^{*}(v) \leq y_{n}^{*}\left(y_{n}\right)$ we have

$$
\left|x_{n}^{*}(u)-x_{n}^{*}(v)\right| \leq c_{n}\left(y_{n}^{*}\left(y_{n}\right)-y_{n}^{*}\left(x_{n}\right)\right) .
$$

For simplicity we use the notation

$$
\begin{aligned}
u_{n}(x) & =x_{n}^{*}(x)-x_{n}^{*}\left(x_{n}\right), \\
v_{n}(x) & =y_{n}^{*}(x)-y_{n}^{*}\left(x_{n}\right), \\
\varphi_{n}(x) & =\left(u_{n}(x), v_{n}(x)\right) \in \mathbb{R}^{2}, \\
\alpha_{n} & =c_{n}\left(y_{n}^{*}\left(y_{n}\right)-y_{n}^{*}\left(x_{n}\right)\right), \\
\eta_{n} & =\frac{y_{n}^{*}\left(y_{n}\right)-y_{n}^{*}\left(x_{n}\right)}{2} .
\end{aligned}
$$

Using this notation, we know that $\eta_{n}>0$ and

$$
u_{n}\left(x_{n}\right)=v_{n}\left(x_{n}\right)=0, \quad v_{n}\left(y_{n}\right)=2 \eta_{n}, \quad \alpha_{n}=2 c_{n} \eta_{n},
$$

and for all $x \in K$,

$$
0 \leq v_{n}(x) \leq 2 \eta_{n} \Rightarrow-\alpha_{n} \leq u_{n}(x) \leq \alpha_{n}
$$


If $K$ is bounded, then $\alpha_{n}=2 c_{n} \eta_{n} \leq c_{n} \operatorname{diam} K$, and so $\alpha_{n} \rightarrow 0$. Moreover, since $v_{n}\left(x_{n}\right)=0$ and $v_{n}\left(y_{n}\right)=2 \eta_{n}$, for each $n$ there exists $x \in K$ with $v_{n}(x)=\eta_{n}$. Let $K$ be unbounded; since $u_{n}$ and $v_{n}$ are both affine, $\varphi_{n}(K)$ must be convex, and as $\varphi_{n}\left(x_{n}\right)=(0,0) \in \varphi_{n}(K)$, it is clear that $(\star)$ remains true if we replace $\alpha_{n}, \eta_{n}$ by $N \alpha_{n}, N \eta_{n}$ with any $N \geq 1$. In this way we can ensure that $\alpha_{n} \rightarrow \infty$ and $\left\|x_{n}\right\| / \alpha_{n} \rightarrow 0$. As we noticed after the proof that (iii) $\Rightarrow$ (ii), we can assume that $\sup _{x \in K} v_{n}(x)=\infty$. Hence, also in this case, there is an $x \in K$ for which $v_{n}(x)=\eta_{n}$. Thus we obtain the following lemma:

Lemma 10. Suppose that condition (iii) of Theorem 4 fails. Then for every constant $c>0$ there is a sequence of positive numbers $c_{n}$ with $c_{n} \rightarrow 0$ as $n \rightarrow \infty$ and there are sequences of positive numbers $\alpha_{n}, \eta_{n}$ such that:

(a) $0 \leq v_{n}(x) \leq 2 \eta_{n} \Rightarrow-\alpha_{n} \leq u_{n}(x) \leq \alpha_{n}$ for all $x \in K$;

(b) $\alpha_{n}=2 c_{n} \eta_{n}$;

(c) there is an $x \in K$ for which $v_{n}(x)=\eta_{n}$;

(d) at least one of the following is satisfied:

(d1) $\alpha_{n} \rightarrow 0$,

(d2) $c \alpha_{n}-\left\|x_{n}\right\| \rightarrow \infty$.

In order to complete the proof that (i) $\Rightarrow$ (iii), it suffices to prove the following lemma:

Lemma 11. Assume that for every $c>0$ there are sequences of positive numbers $\alpha_{n}, \eta_{n}, c_{n}$ with $c_{n} \rightarrow 0$ for which conditions (a)-(d) of Lemma 10 are satisfied. Then for every $0<\varepsilon<1, \delta>0$ and $L>1$ there are continuous functions $F, G: K \rightarrow \mathbb{R}$ such that:

- $G+F$ and $G-F$ are convex;

- $\operatorname{Lip}(G) \leq \varepsilon$

- $2 L \geq \operatorname{Lip}(F) \geq L ;$ and

- either (d1) holds and $\sup |F| \leq \delta$, or (d2) holds and $F(x)=0$ for every $\|x\| \leq \delta$.

Indeed, by Lemma 11, for any sequences $\varepsilon_{n} \rightarrow 0, L_{n} \rightarrow \infty$ and $\delta_{n}>0$ we can find $F_{n}, G_{n}: K \rightarrow \mathbb{R}$ and points $a_{n}, b_{n} \in K$ such that $G_{n} \pm F_{n}$ are convex, $\operatorname{Lip}\left(G_{n}\right) \leq \varepsilon_{n}, \operatorname{Lip}\left(F_{n}\right) \leq 2 L_{n},\left|F_{n}\left(b_{n}\right)-F_{n}\left(a_{n}\right)\right|>L_{n}\left\|b_{n}-a_{n}\right\|$, and either (d1) holds and $\sup \left|F_{n}\right| \leq \delta_{n}$, or (d2) holds and $F_{n}(x)=0$ for every $\|x\| \leq \delta_{n}$. We can also assume that for a fixed point $x^{0} \in K, G_{n}\left(x^{0}\right)=0$ for every $n$. Then, by choosing $\sum_{n} \varepsilon_{n}<\infty, g=\sum_{n} G_{n}$ is Lipschitz. By requiring $\sum_{n} \delta_{n}<\infty$ in case (d1) and $\delta_{n} \rightarrow \infty$ in case (d2), we can also ensure that $f=\sum_{n} F_{n}$ exists and is continuous. Since the sum of convex functions is convex, $g+f$ and $g-f$ are convex. If we also require that $\sum_{i<n} 2 L_{i}<L_{n} / 3$ for every $n$, and $\sum_{i>n} 2 \delta_{i}<\left(L_{n} / 3\right)\left\|b_{n}-a_{n}\right\|$ for every $n$ 
if (d1) holds, or $\delta_{i}>\max \left(\left\|a_{n}\right\|,\left\|b_{n}\right\|\right)$ for every $i>n$ if (d2) holds, then, in the first case,

$$
\left|f\left(b_{n}\right)-f\left(a_{n}\right)\right|>L_{n}\left\|b_{n}-a_{n}\right\|-\sum_{i<n} 2 L_{i}\left\|b_{n}-a_{n}\right\|-2 \sum_{i>n} \delta_{i} \geq \frac{L_{n}}{3}\left\|b_{n}-a_{n}\right\|,
$$

and in the second case,

$$
\begin{aligned}
\left|f\left(b_{n}\right)-f\left(a_{n}\right)\right| & =\left|\sum_{i=1}^{n} F_{i}\left(b_{n}\right)-F_{i}\left(a_{n}\right)\right|>L_{n} \mid b_{n}-a_{n}\left\|-\sum_{i<n} 2 L_{i}\right\| b_{n}-a_{n} \| \\
& >\frac{2 L_{n}}{3}\left\|b_{n}-a_{n}\right\| .
\end{aligned}
$$

In either case, we deduce that $f$ is not Lipschitz and (i) $\Rightarrow($ iii), as required.

Before passing to the proof of Lemma 11, we prove the following simple result. This is the only place in our proof at which the assumption $0 \in K$ is crucial, since the statement is clearly false for the set $\{1\} \times \mathbb{R} \subset \mathbb{R}^{2}$.

Lemma 12. For all $\eta, \varrho>0, x \in K$ and $w \in S_{X}$ there are $y, z \in$ $B(x, \varrho) \cap K$ such that

$$
\left\|\frac{y-z}{\|y-z\|}-w\right\|<\eta
$$

Proof. Since for all $u, v$ in $B(0, \varrho) \cap K$ and $x \in K$ the vectors

$$
u^{\prime}=\frac{\varrho}{\|x\|+\varrho} u+\frac{\|x\|}{\|x\|+\varrho} x \quad \text { and } \quad v^{\prime}=\frac{\varrho}{\|x\|+\varrho} v+\frac{\|x\|}{\|x\|+\varrho} x
$$

are in $B(x, \varrho) \cap K$ and $\left(u^{\prime}-v^{\prime}\right) /\left\|u^{\prime}-v^{\prime}\right\|=(u-v) /\|u-v\|$, it is enough to prove the lemma for $x=0$.

Since $\overline{\operatorname{span}}(K)=X$, there are positive numbers $a_{1}, \ldots, a_{n}, b_{1}, \ldots, b_{m}$ and there are $u_{1}, \ldots, u_{n}, v_{1}, \ldots, v_{m} \in K$ such that

$$
\left\|\frac{\sum_{i=1}^{n} a_{i} u_{i}-\sum_{j=1}^{m} b_{j} v_{j}}{\left\|\sum_{i=1}^{n} a_{i} u_{i}-\sum_{j=1}^{m} b_{j} v_{j}\right\|}-w\right\|<\eta .
$$

Then for $M=\max \left(\sum_{i=1}^{n} a_{i}, \sum_{j=1}^{m} b_{j}\right)$, the vectors $u=\sum_{i=1}^{n} a_{i} u_{i} / M$ and $\sum_{j=1}^{m} b_{j} v_{j} / M$ are in $K$, and satisfy the claim of the lemma.

Proof of Lemma 11. Set

$$
c=\frac{1}{(\varepsilon / 80)^{2}},
$$

and choose $n$ to be so large that

$$
c_{n}<\frac{(\varepsilon / 80)^{2}}{2 e^{L+2 \varepsilon}} .
$$


In addition, in case (d1), for $n$ large enough we know that

$$
\alpha_{n}<\frac{\delta}{L+2 \varepsilon},
$$

and in case (d2), for $n$ large enough,

$$
\frac{\alpha_{n}}{(\varepsilon / 80)^{2}}-\left\|x_{n}\right\|>\delta
$$

Fix an $n$ for which these inequalities are satisfied, and set $\eta=\eta_{n}, \alpha=\alpha_{n}$,

$$
\beta=\frac{\alpha_{n}}{(\varepsilon / 80)^{2}}, \quad \gamma=e^{L+2 \varepsilon} \beta<\eta,
$$

and $u(x)=u_{n}(x), v(x)=v_{n}(x), \varphi(x)=(u(x), v(x))$. Let $f$ and $g$ be the functions given by Lemma 9 when the parameters are $\varepsilon / 80, L+2 \varepsilon, \alpha, \beta$ and $\gamma$. Define

$$
F(x)=f(\varphi(x)), \quad G(x)=g(\varphi(x)) .
$$

Since $\gamma<\eta$, from (a) of Lemma 10 we can see

$$
\varphi(K) \subset([-\alpha, \alpha] \times \mathbb{R}) \cup(\mathbb{R} \times(\mathbb{R} \backslash[0,2 \gamma])) .
$$

Thus $G+F$ and $G-F$ are continuous convex functions. We also know from (C) of Lemma 9 that if (d1) holds, then $\sup |F| \leq \alpha(L+2 \varepsilon)<\delta$, and if (d2) holds, any $x$ with $\|x\| \leq \delta$ satisfies $v(x) \leq\|x\|+\left\|x_{n}\right\| \leq \delta+\left\|x_{n}\right\|<\beta$ and hence $F(x)=0$ by (C). We also see from (B), (D) of Lemma 9 that $\operatorname{Lip}(g)<\varepsilon$ and $\operatorname{Lip}(F) \leq \operatorname{Lip}(f) \leq \sqrt{L^{2}+\varepsilon^{2}} \leq 2 L$.

By continuity and the fact that $\gamma<\eta$, by applying (c) of Lemma 10 we can find an $x=x^{0}$ for which $v\left(x^{0}\right)=\gamma$. By Lemma 12, for every $\varrho>0$ and $\vartheta<1$ we can find $y, z \in B\left(x^{0}, \varrho\right) \cap K$ for which $x_{n}^{*}(y-z)>(1-\vartheta)\|y-z\|$. If $\varrho$ is small enough then $v(y)$ and $v(z)$ are close to $v\left(x^{0}\right)=\gamma$. From (B) of Lemma 9 we get

$$
|f(u(z), v(y))-f(u(z), v(z))|<(\varepsilon / 80)|v(y)-v(z)|<(\varepsilon / 2)\|y-z\|,
$$

and if $\varrho$ is small enough then (D) of Lemma 9 gives

$$
\begin{aligned}
|f(u(y), v(y))-f(u(z), v(y))| & >((L+2 \varepsilon)-\varepsilon)|u(y)-u(z)| \\
& =(L+\varepsilon)\left|x_{n}^{*}(y)-x_{n}^{*}(z)\right| \\
& \geq(L+\varepsilon)(1-\vartheta)\|y-z\| .
\end{aligned}
$$

Therefore

$$
\begin{aligned}
|F(y)-F(z)|= & |f(u(y), v(y))-f(u(z), v(z))| \\
\geq & |f(u(y), v(y))-f(u(z), v(y))| \\
& -|f(u(z), v(y))-f(u(z), v(z))| \\
\geq & ((L+\varepsilon)(1-\vartheta)-\varepsilon / 2)\|y-z\| .
\end{aligned}
$$

For $\vartheta$ small enough this gives $\operatorname{Lip}(F)>L$, and the lemma is proved. 
REMARK 13. Let $X=\ell_{1}$, and let $e_{1}, e_{2}, \ldots$ be the standard basis of $\ell_{1}$. Let

$$
\begin{aligned}
C_{0} & =\left\{\sum_{j} \lambda_{j} e_{j} \in \ell_{1}: 0 \leq \lambda_{j} \leq \frac{\lambda_{1}}{j}\right\}, \\
C_{n} & =\left\{\sum_{j} \lambda_{j} e_{j} \in \ell_{1}: \lambda_{j} \geq 0, \sum_{j} \lambda_{j} \leq 2 \lambda_{n} \leq 2 n\right\}, \\
D & =\left\{\sum_{n=1}^{\infty} a_{n}: a_{n} \in C_{n}, \sum_{n=1}^{\infty} a_{n} \in \ell_{1}\right\}, \\
K_{0} & =\left\{x \in \ell_{1}: d\left(x, C_{0}\right)<1\right\}, \\
K_{1} & =\left\{x \in \ell_{1}: d(x, D)<1\right\} .
\end{aligned}
$$

It is easy to see that $K_{0}$ and $K_{1}$ are open, unbounded convex sets containing the origin, with $\overline{\operatorname{span}}\left(C_{0}\right)=X, C_{0} \subset K_{0}$ and $\overline{\operatorname{span}}\left(K_{1}\right)=X$. However, $K_{1}$ does not contain any half-line, and conditions (i)-(iii) of Theorem 4 fail for $K_{0}$, but hold for $K_{1}$.

Proof. It is easy to check that for every $u=\sum \lambda_{j} e_{j} \in B(0, n+1) \cap C_{0}$ we have $0 \leq \lambda_{n} \leq 1$, and then $B(0, n+1) \cap K_{0}$ does not contain any $u, v$ with $u-v=4 e_{n}$, which contradicts (ii) for $K_{0}$.

For all $x=d+z \in K_{1}, d \in D,\|z\|<1, r>0, n>\max (\|d\|, r)$ and $y=\sum_{j} y_{j} e_{j} \in S_{X}$ we have

$$
2 n e_{4 n}+d+n \sum_{\left\{j: y_{j} \geq 0\right\}} y_{j} e_{j} \in C_{4 n}, \quad 2 n e_{4 n}+d-n \sum_{\left\{j: y_{j}<0\right\}} y_{j} e_{j} \in C_{4 n},
$$

therefore

$$
\begin{aligned}
& u=2 n e_{4 n}+x+n \sum_{\left\{j: y_{j} \geq 0\right\}} y_{j} e_{j} \in K_{1} \cap B(x, 3 n), \\
& v=2 n e_{4 n}+x-n \sum_{\left\{j: y_{j}<0\right\}} y_{j} e_{j} \in K_{1} \cap B(x, 3 n),
\end{aligned}
$$

and $u-v=n y$. Then $u^{\prime}=x+(r / n)(u-x)$ and $v^{\prime}=x+(r / n)(v-x)$ are both in $K_{1} \cap B(x, 3 r)$ and $u^{\prime}-v^{\prime}=r y$. That is, (ii) is satisfied for $K_{1}$ with $c=1 / 3$.

It only remains to check that $K_{1}$ does not contain any half-line. Assume instead that $K_{1}$ contains a half-line of direction $y=\sum y_{n} e_{n} \in S_{X}$. It is clear that $y_{n} \geq 0$ for every $n$. Choose an $N \geq 4$ for which

$$
\sum_{n=1}^{N-1} y_{n}>\frac{2}{3} \text { and } \sum_{n=N}^{\infty} y_{n}<\frac{1}{3} .
$$

For every $x \in K_{1}$ and $\lambda \geq 0$, we have $x+\lambda y \in K_{1}$. Indeed, fix some $v \in K_{1}$ such that $v+[0, \infty) y \subset K_{1}$. Since $K_{1}$ is open, there is an $\varepsilon>0$ such that 
$x+\varepsilon(x-v) \in K_{1}$. By convexity,

$$
x+\lambda y=\frac{1}{1+\varepsilon}(x+\varepsilon(x-v))+\frac{\varepsilon}{1+\varepsilon}\left(v+\frac{1+\varepsilon}{\varepsilon} \cdot \lambda y\right) \in K_{1} .
$$

In particular, $4 N^{2} y \in K_{1}$, and so $4 N^{2} \sum_{n} y_{n} e_{n}=\sum_{n} a_{n}+b$, where $a_{n}=$ $\sum_{j} \lambda_{j n} e_{j} \in C_{n}$ for every $n$ and $b=\sum_{j} b_{j} e_{j} \in B(0,1)$. We also have $\lambda_{j n} \geq 0$ for every $j$ and $n$, and $\sum_{j, n} \lambda_{j n}<\infty$. Since $C_{n} \subset B(0,2 n)$, we obtain

$$
\sum_{n=1}^{N-1} \sum_{j=1}^{N-1} \lambda_{j n} \leq \sum_{n=1}^{N-1} 2 n<N^{2}
$$

Hence

$$
\begin{aligned}
4 N^{2}\left(\sum_{j=1}^{N-1} y_{j}-\sum_{j=N}^{\infty} y_{j}\right) & =\sum_{n=1}^{\infty}\left(\sum_{j=1}^{N-1} \lambda_{j n}-\sum_{j=N}^{\infty} \lambda_{j n}\right)+\sum_{j=1}^{N-1} b_{j}-\sum_{j=N}^{\infty} b_{j} \\
& \leq N^{2}-\sum_{n=N}^{\infty}\left(\sum_{j=N}^{\infty} \lambda_{j n}-\sum_{j=1}^{N-1} \lambda_{j n}\right)+\sum_{j=1}^{N-1} b_{j}-\sum_{j=N}^{\infty} b_{j},
\end{aligned}
$$

where for every $n \geq N$ we have

$$
\sum_{j=N}^{\infty} \lambda_{j n}-\sum_{j=1}^{N-1} \lambda_{j n}=2 \sum_{j=N}^{\infty} \lambda_{j n}-\sum_{j=1}^{\infty} \lambda_{j n} \geq 2 \sum_{j=N}^{\infty} \lambda_{j n}-2 \lambda_{n n} \geq 0
$$

and

$$
\sum_{j=1}^{N-1} b_{j}-\sum_{j=N}^{\infty} b_{j} \leq 1
$$

Therefore

$$
4 N^{2}\left(\sum_{j=1}^{N-1} y_{j}-\sum_{j=N}^{\infty} y_{j}\right) \leq N^{2}+1 .
$$

This is a contradiction, since $\sum_{j=1}^{N-1} y_{j}-\sum_{j=N}^{\infty} y_{j}>1 / 3$.

REMARK 14. Note that it does not make any difference if, instead of (i), we assume that for some (or every) $k \geq 2$, and for any convex functions $h_{1}, \ldots, h_{k}$, the functions must be Lipschitz whenever their sum is Lipschitz.

Acknowledgments. This work has been carried out while the second named author was visiting the Mathematics Department of the University College London. Both authors would like to express their sincere gratitude to Toby C. O'Neil for carefully reading the manuscript and making many invaluable corrections and suggestions. 


\section{References}

[BL] Y. Benyamini and J. Lindenstrauss, Geometric Nonlinear Functional Analysis, Vol. 1, Amer. Math. Soc. Colloq. Publ. 48, Amer. Math. Soc., Providence, RI, 2000 .

[C1] M. Cepedello-Boiso, Approximation of Lipschitz functions by $\Delta$-convex functions in Banach spaces, Israel J. Math. 106 (1998), 265-284.

[C2] - On regularization in superreflexive Banach spaces by infimal convolution formulas, Studia Math. 129 (1998), 265-284.

[DVZ] J. Duda, L. Veselý and L. Zajíček, On D.C. functions and mappings, preprint, 2001.

[VZ] L. Veselý and L. Zajíček, Delta-convex mappings between Banach spaces and applications, Dissertationes Math. (Rozprawy Mat.) 289 (1989).

Department of Mathematics

University College London

Gower Street

London, WC1E 6BT, United Kingdom

E-mail: mari@math.ucl.ac.uk
Department of Mathematics Hebrew University, Givaat-Ram Jerusalem, Israel E-mail: naor@math.huji.ac.il

Received July 22, 2002

Revised version April 9, 2003 